\title{
Article
}

\section{Two-Parameter CFAR Ship Detection Algorithm Based on Rayleigh Distribution in SAR Images}

\author{
Ruochen $W^{1, *}$ (1)
}

1 CommSensLab, Department of Signal Theory and Communications, Universitat Politècnica de Catalunya (UPC), Campus Nord, Carrer de Jordi Girona, 1, 3, 08034 Barcelona, Spain; ruochen.wu@upc.edu

* Correspondence: ruochen.wu@upc.edu

\begin{abstract}
Synthetic Aperture Radar (SAR) is an active type of microwave remote sensing. Using the microwave imaging system, remote sensing monitoring of the land and global ocean can be done in any weather conditions around the clock. Detection of SAR image targets is one of the main needs of radar image interpretation applications. In this paper, an improved two-parameter CFAR algorithm based on Rayleigh distribution and morphological processing is proposed to perform ship detection and recognition in high resolution SAR images. Through simulation experiments, comprehensive study of the two algorithms for high resolution SAR image target detection is achieved. Finally, the results of ship detection experiments are compared and analyzed, and the effects of detection are evaluated according to the Rayleigh distribution model and algorithms.
\end{abstract}

Keywords: SAR; Ship detecion; CFAR; Mathematical morphology

\section{Introduction}

SAR is a high-resolution microwave imaging radar. Compared with optical remote sensing, its image is not restricted by environmental factors such as weather and light, and it can detect and identify objects of interest all the time and in all weather [1-4]. In military use, SAR is used primarily in the fields of target recognition, battlefield surveillance, and precision targeting; in civil use, it is mainly used in fields such as earth monitoring, disaster warning and marine environment monitoring $[5,6]$. With the continuous development of SAR technology, the detection and subsequent identification of ground targets (such as vehicles, ships, special buildings) have a very important value.

Today's technology in the field of SAR image target recognition is becoming more mature. Researchers have systematically analyzed SAR target segmentation and explained algorithms of different technical levels, such as Constant False Alarm Rate (CFAR) segmentation technology, MRF-based target segmentation and Maxflow [7]. The theory of computer vision graphics is used for image segmentation and the OTSU algorithm is used to achieve automatic image segmentation. For target detection, CFAR algorithm, Beamlet-based SAR image target detection method, feature value extraction by two-dimensional main component analysis and many other solutions have been studied mainly, and they have achieved well expected results [8]. For the most important part of target segmentation and target detection in SAR image target recognition, researchers have developed the algorithms with the highest recognition rates, and their average recognition rates have remained above $90 \%$. CFAR is the deepest, most widely used, and best-effective type of many target detection algorithms. This algorithm was proposed by Wackerman C. et al. in 2001 based on the Ottawa Defense Research Center's ship target detection investigation [9]. Its core is to establish a distribution model based on local area data, draw the probability density curve of the model, and then calculate the object's pixel segmentation threshold using the false alarm rate. Finally, the target with a high gray value in the SAR image is detected by threshold. To this end, this paper proposes an improved two-parameter CFAR algorithm based on the Rayleigh 
distribution model, to suppress background noise by adjusting for sea noise in the SAR image. Generally, the probability density function of sea clutter and a small amount of noise tends to deviate from the Rayleigh distribution, but there are still some clutter backgrounds with significant non-Rayleigh characteristics. The non-Rayleigh clutter background is different from the Rayleigh clutter background, containing the scale and shape parameters. In the real mess environment, these two parameters are often unknown, so this paper adopts the two-parameter CFAR detection algorithm.

Nevertheless, current research on SAR image target recognition is mainly focused on performing basic algorithms and improving the recognition rate. There is no sufficient research on SAR image target recognition suitable for various complex backgrounds and reduction of detection target distortion. These problems need to be solved through actual work. Therefore, this paper uses mathematical morphology (MM), an image analysis subject based on lattice theory and topology. It is the basic theory of mathematical morphology image processing and can be used in areas such as noise suppression and image segmentation [10]. Its basic operations include Erosion and Dilation, Opening and Closing operations. Due to factors such as an unreasonable setting of the false alarm rate or an incorrect setting of the background disorder, certain false alarms will occur in the target detection process. Some pixels whose sizes do not meet the target parameters are also identified as targets. In these cases, morphological operations can be used to remove them. For the filtered SAR image, a simple eroding operation can remove small nonsensical objects in the image and speckle noises with independent high brightness that is erroneously judged as the target pixel. The simple dilation operation can fill the black hole caused by the low value speckle noises in the target area and the missing target pixels, and can connect the adjacent unconnected target area pixels.

\section{Dateset and Methods}

\subsection{SAR dataset}

This paper uses the SAR imaging ship detection dataset first published by researcher Chao Wang's team at the Key Laboratory of Digital Earth Science, Institute of Remote Sensing and Digital Earth, Chinese Academy of Sciences. This dataset comes from SAR images from multiple sources and multiple modes.The data is based on SAR data from China Ganfen(GF)-3 and Sentinel-1.

The GF-3 image modes are Strip-Map (UFS), Fine Strip-Map 1 (FSI), Full Polarization 1 (QPSI), Full Polarization 2 (QPSII), and Fine Strip-Map 2 (FSII). The resolutions of these five image models are $3 \mathrm{~m}, 5 \mathrm{~m}, 8 \mathrm{~m}, 25 \mathrm{~m}$ and $10 \mathrm{~m}$. Sentinel-1's image modes are band (S3 and S6) and wide-format image [11].

Experimental data is collected from more than 40,000 SAR images in the SAR dataset mentioned above. According to the uniform background (stable state of the sea), the noise background, the land and marine background (coast or port), they are divided into three groups. Several satellite images are randomly selected by each, including image data from GF-3 and Sentinel-1A. Figure 1 shows some example images of the SAR dataset used in experiments. The initial data is all cropped images that have been cropped from high resolution SAR images, and each size is $256 \times 256$ pixels. The background of the images contains a variety of scenes such as the ocean, coast, land and sea to test whether CFAR and MM methods have good ship detection results. To make the experiment more rigorous, this paper uses the metadata in the dataset produced by Chao Wang's team to specify the number of ships in the SAR (Aerospace Information Research Institute, Chinese Academy of Sciences) images. Each cut image corresponds to a meta file, which will make the final verification job convenient and efficient, as shown in Figure 2. 


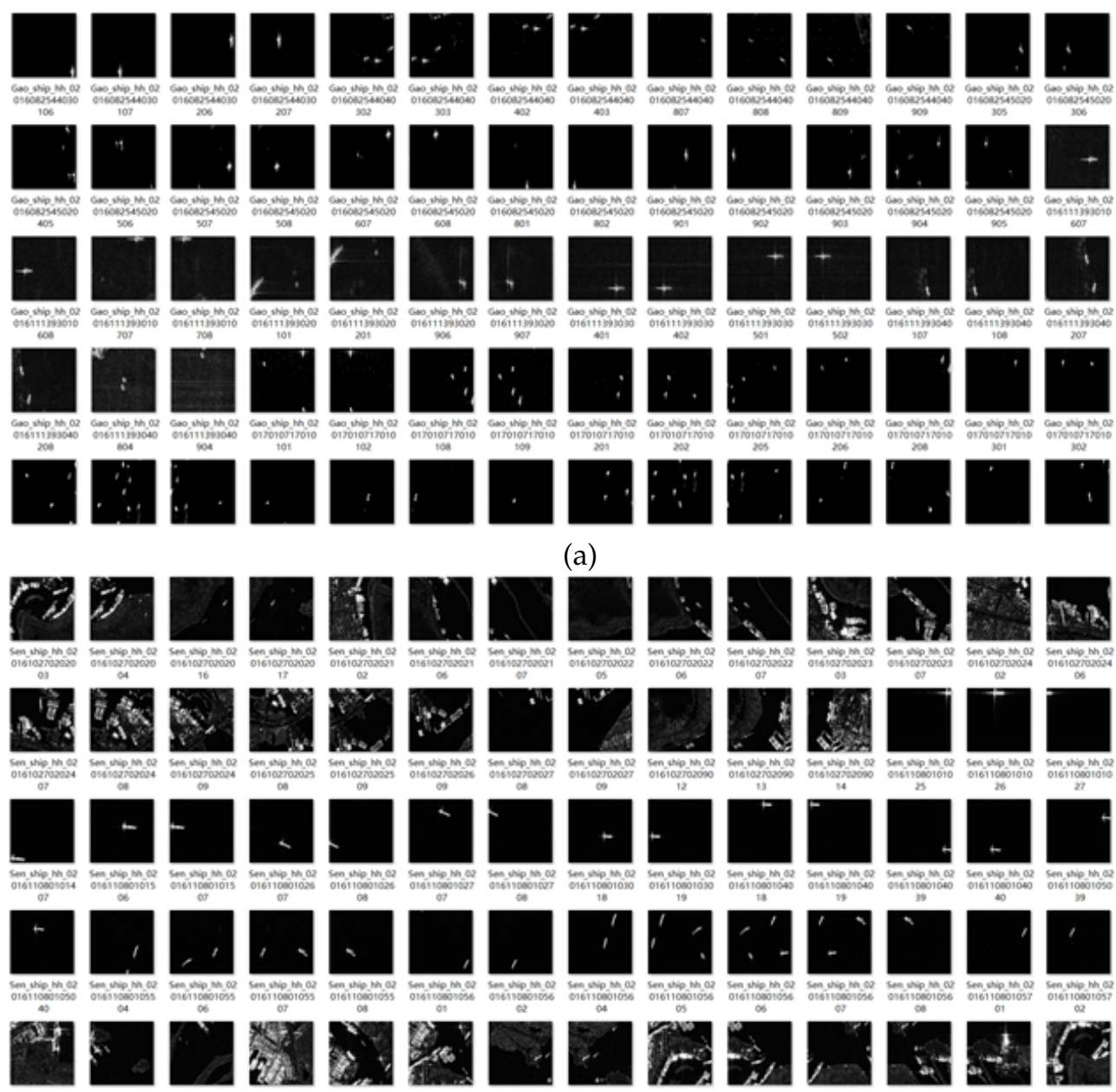

(b)

Figure 1. Some examples of ship chips. The group (a) is the cropped ship chips from the Gaofen-3 images. The group (b) is the cropped ship chips from the Sentinel-1A images.
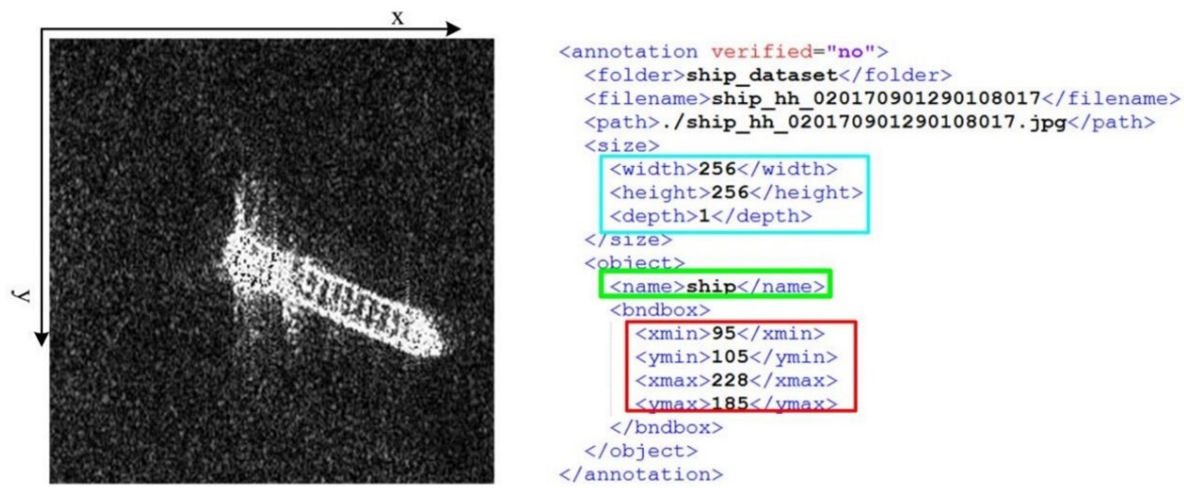

Figure 2. Detailed information of a labeled ship chip shown in [11]. The red, green, and cyan rectangles indicate the ship location, the object name, and the ship chip shape in the image on the left.

\subsection{Target detection in CFAR-based SAR images}

CFAR is a pixel-level target detection algorithm. For targets such as vehicles, ships, and aircrafts, they are generally required to have a strong contrast to the background clutter. Target detection is achieved by judging whether the gray value of each pixel exceeds a certain preset value [12]. In this case, the detection threshold is generally related to the false alarm rate, the statistical pattern of the background disorder around 
the target and the CFAR detector. Given the false alarm probability and the distribution probability density of the background disorder, it can be obtained

$$
P_{f a}=\int_{T}^{\infty} p(x) d x
$$

where $x$ is the gray value of the detected pixel and $T$ is the detection threshold.

According to Equation (1), when $x>T$, the detected pixel is the target itself; when $x \leq T$, the detected pixel is the background.

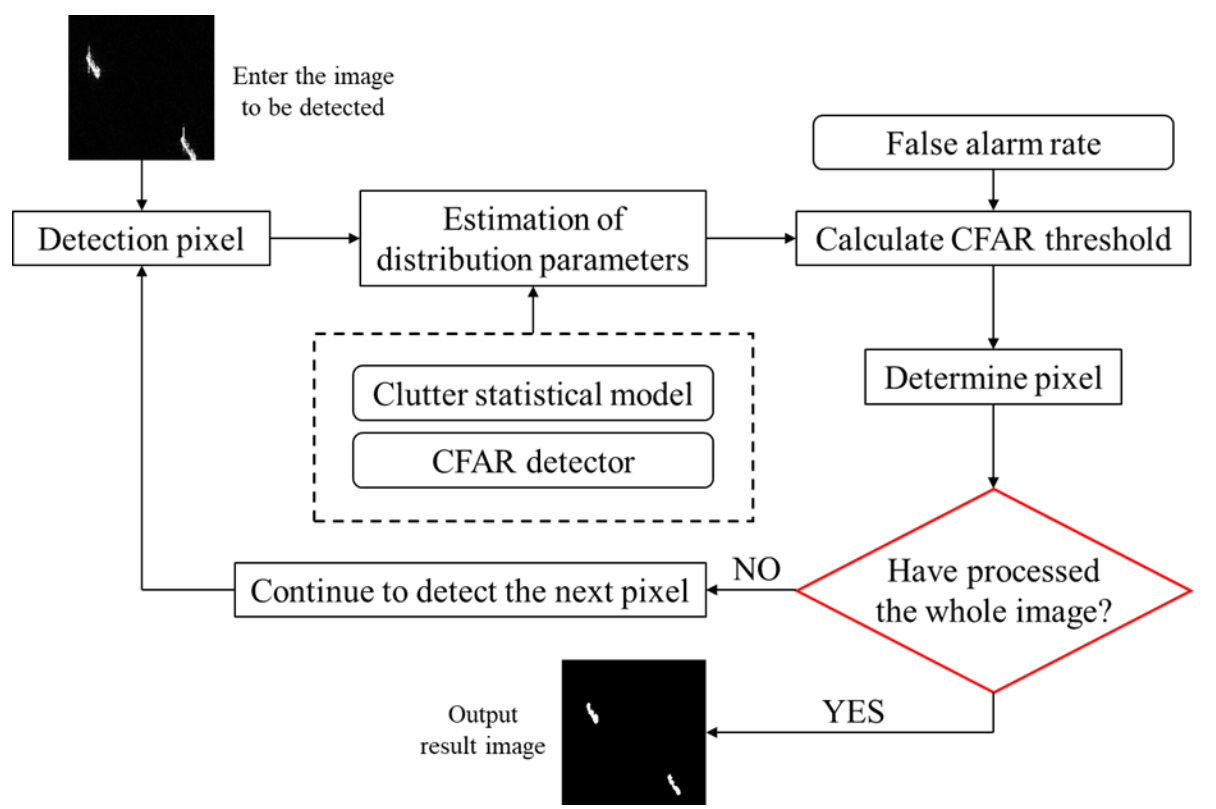

Figure 3. General flow of the CFAR algorithm.

Figure 3 shows the general flow of the CFAR detection algorithm. The Clutter statistical model, CFAR detector and False alarm rate are the three default elements of the CFAR detection algorithm. After determining the above items, the CFAR threshold can be calculated, and then the gray value of the detected pixel is compared with the threshold to determine whether the test pixel is target or background. In this way, each pixel in the image is detected and considered to complete the CFAR detection of the target. In general, the CFAR detection algorithm consists of importing a SAR image and discerning each pixel in the entire image. Determine the background distribution of the clutter based on the SAR image and then estimate the distribution parameters of the clutter pixels in the sliding window. In the case of a given false alarm probability, the CFAR threshold is resolved and compared with the corresponding pixel value to obtain the pixel detection result. This process will work with all the pixels in the input SAR image, eventually traversing the entire image to get the detection result.

The SAR image itself features a lot of speckled noise and has a low signal-to-noise ratio. Typically, prior knowledge of the background and targets is difficult to obtain, so are random changes in the background environment and complex background texture information, so it is very important to use statistical models for target detection. The clutter statistical model for SAR images counts on statistical methods to describe SAR imaging data [13]. At present, the most widely used clutter statistical models mainly include the Rayleigh distribution model, the Weibull distribution model, the Pearson distribution model, the Gamma distribution model, and the $G^{0}$ distribution model.

The Rayleigh distribution is the most common type of distribution used to describe the time-varying statistical characteristics of the flat-fading signal receiving envelope or independent multipath component receiving envelope. The envelope of the sum of two orthogonal Gaussian noise signals obeys the Rayleigh distribution. As the basis for 
the study of SAR image clutter statistical models, it is a multiplicative noise one which has been widely used in processing, analyzing, and modeling of SAR images [14]. Its probability density function is:

$$
f(x ; \sigma)=\frac{x}{\sigma^{2}} e^{-x^{2} / 2 \sigma^{2}}, x \geq 0
$$

where $\sigma$ is the distribution parameter, which can be calculated using the Rayleigh distribution formula shown in Equation (3).

$$
\sigma=\frac{2 \mu^{2}}{x}
$$

where $\mu$ is the mean.

Figure 4 shows the Rayleigh probability distribution curve at different $\sigma$.

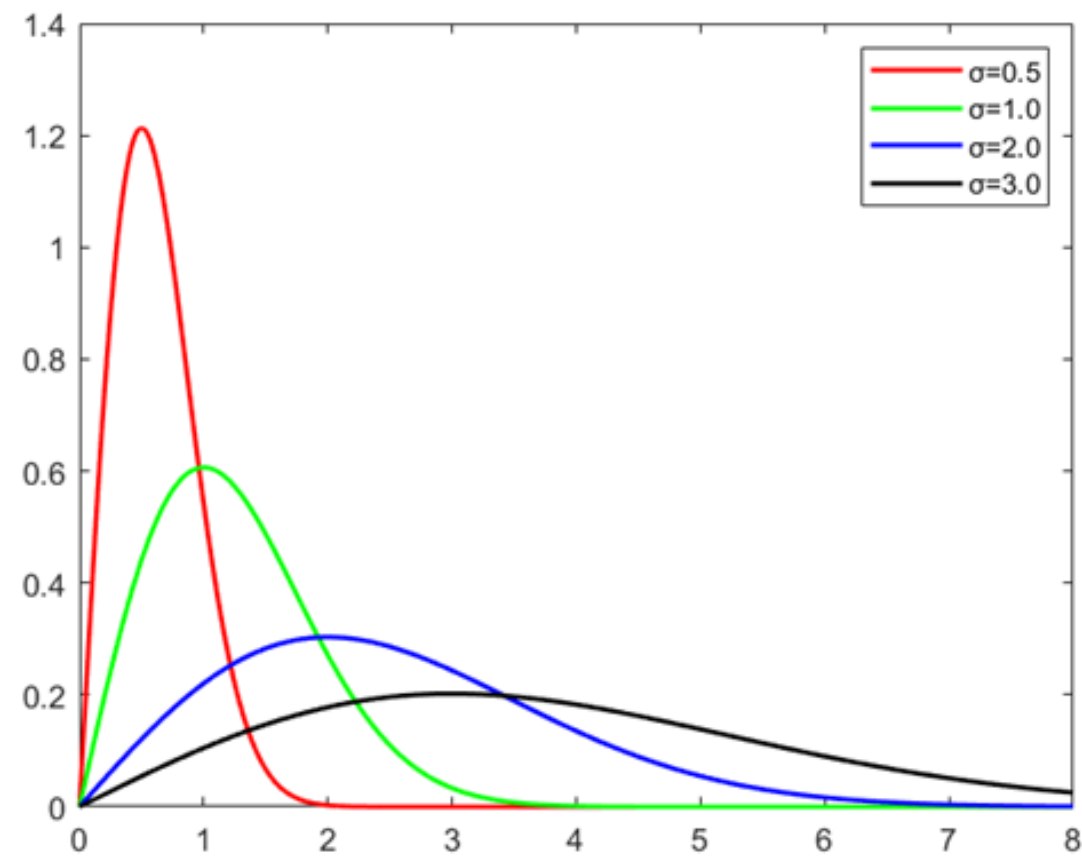

Figure 4. Diagram of Rayleigh distribution probability density obtained by MATLAB R2021b simulation.

For the detection of ship targets, the Rayleigh distribution is usually used in the gray value statistics from sea clutter images. However, in high resolution SAR images, Rayleigh distribution may not accurately match the gray value of sea clutter; and in complex cases, the fitting between the true distribution of gray value and what is relatively poor. Thus, it is necessary to conduct experiments in various types of situations.

The purpose of CFAR radar detection is to keep the false alarm rate during the detection process at a relatively constant level. The CFAR detector traverses all pixels in the complete SAR image through a sliding window to achieve the purpose of detecting SAR image targets. The commonly used CFAR sliding window is a hollow one, consisting of three parts: the target zone, the protection zone, and the clutter zone [15]. CFAR can be divided into global and local CFAR algorithms according to window size [16]. If the size is smaller than that of the SAR image, it will be a local CFAR algorithm (Figure 5). The local CFAR algorithm continuously realizes background clutter data modeling, and local threshold calculation and comparison. Its advantage concludes more accurate solution of the threshold and model distribution parameters as well as better detection 
performance, which appears suitable for detecting SAR image targets with complex and uneven scenes.

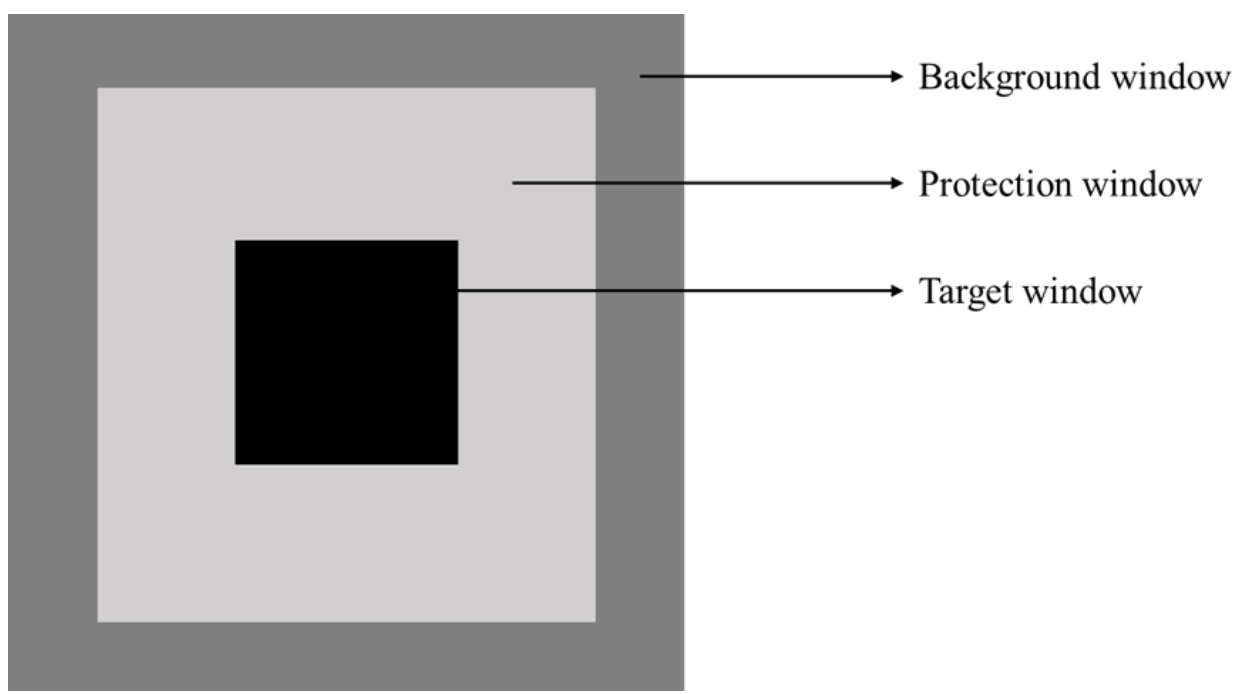

Figure 5. Local CFAR detection window. It defines two sliding windows: protection window and background window. The background window is for background clutter statistics to calculate the target detection threshold; while the protection window is for preventing the target pixels from leaking into the background window, thereby affecting the correctness of the background clutter statistics.

When the size of the sliding window equals the size of the SAR image, the global CFAR algorithm is used to all background clutter pixels in the entire image to estimate the background model parameters to find a global threshold, and then compare each pixel in the SAR image with the threshold of the segmented image. The global CFAR algorithm has fast calculation and is suitable for SAR images with a relatively uniform background distribution. While the SAR image scene is more complicated, the false alarm rate of detection result will be higher. The CFAR detection algorithm is generally based on an iterative calculation mode, which quickly calculates the statistics of the background clutter data in the sliding window around the pixel to be detected, and gets the estimated value of the statistics after the iterative calculation. The more the background clutter data overlaps in the adjacent pixel sliding window, the better the effect of the iterative calculation mode.

\subsection{Target detection in SAR images based on morphological operations}

$\mathrm{MM}$ is a technique for the analysis and treatment of geometric structures, based on set theory, lattice theory, topology and random functions. It not only applies to digital images, but can be used for graphics, polygon meshes, solids, and many other spatial structures [17]. The concepts of topology and geometric space, such as size, shape, convexity, connectivity, and geodetic distance introduced by MM in both continuous space and collection space, can be characterized by MM in continuous and discrete spaces. It is also the basis of morphological image processing, consisting of a set of operators that transform images based on previous characteristics. MM was originally developed for binary images and later expanded to functional and grayscale images [10]. In terms of image processing, MM is divided into binary morphology and grayscale morphology, frequently applied to image segmentation, thinning, skeleton extraction, edge extraction, shape analysis, corner detection, watershed algorithms, etc.

The object of morphological operations is a set [18]. Suppose that both the binary image and the structural element are collections of images defined in a two-dimensional Cartesian grid coordinate system. The structural element moves in the coordinate plane and at each pixel position of the image, it performs a specific logical operation on image, 
and the result of the operation is stored at the position corresponding to the pixel in the output image.

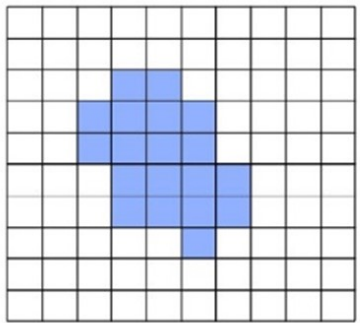

(a)

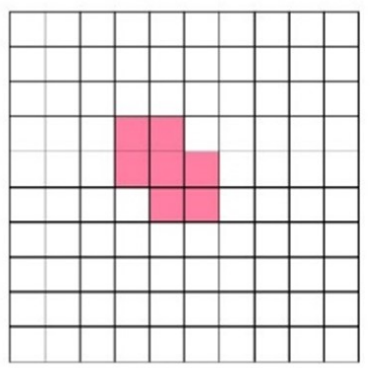

(a)

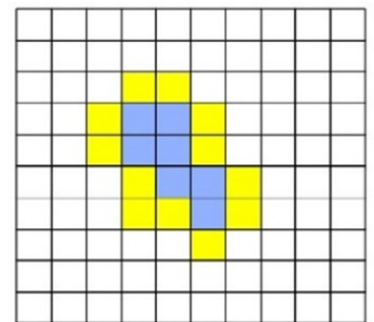

(b)

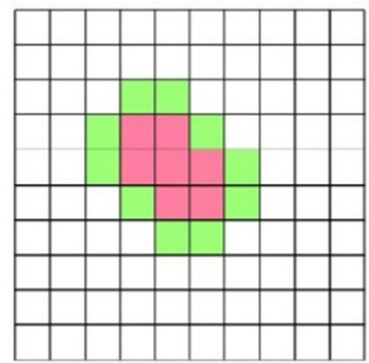

(b)

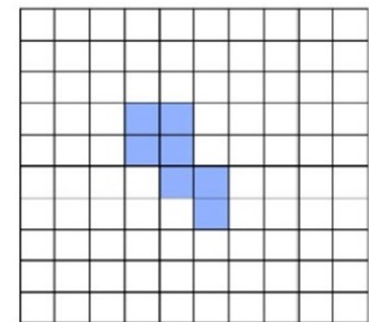

(c)

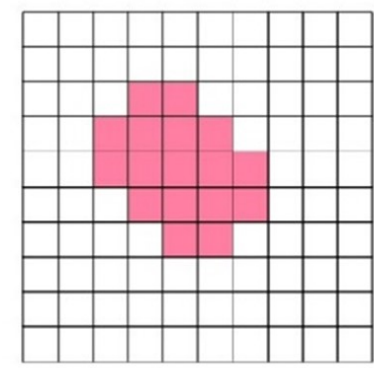

(c)

Figure 6. Schematic diagrams of morphological erosion and dilation operations have been shown in [19]. The pink graphic shows above, erosion consists of marking the pixels at the origin of the sub-image that are the same as the structural elements of the destination image (as in subfigure (a)). The image boundary can be made to shrink to eliminate small, nonsensical objects to obtain (c). For the blue graphic, dilation consists of combining the background points in contact with the target area on the target and expanding the boundary of the target outward (as in subfigure (b)). The function is to fill some gaps in the target area and remove the small particle noise contained in it to obtain (c). In addition, the opening and closing operations are the superposition of the two previous basic operations. The process of first erosion and then dilation is called opening, and the process of first dilation and then erosion is called closing.

Certain false alarms will occur in the target detection process, due to factors such as unreasonable or incorrect setting of the false alarm rate, and complex background disorder. Some pixels whose size does not meet the object parameters are also recognized as a target. In these cases, they can be eliminated by morphological operations. For the filtered SAR image, a simple erosion operation can remove small nonsensical objects and speckle noises with independent high brightness that is erroneously judged as the target pixel in the image. And the dilation operation can fill the black hole caused by the low value speckle noises in the target area, and it can also fill in the missing target pixels and connect the adjacent unconnected target area pixels. Normally, after CFAR detection, there will be some small interfering white noises in the SAR image, which can be eliminated by opening, and the small holes within the target in the CFAR detection result can be filled by closing. On the other hand, after the CFAR detection algorithm detects the SAR image, the morphological operator is used to process the detection result, which can retain large SAR image targets and filter out small interferences. It should be noted that it is unsuitable for opening operation for the ship's target in the SAR image, due to the shape characteristics of the target itself. According to the post-processing needs of the ships' target detection data, the closing operation should be selected.

As a matter of fact, for ship detection, thinning (or Skeletonization) can also be used in morphological operations. This method thins lines with a line width greater 
than 1 pixel in the original image so that they are only one pixel wide, forming a "skeleton", which is convenient for image analysis, such as image feature extraction. It is a basic problem to find the refined structure of binary images in the field of image recognition. Also called Median Axis Transformation, skeleton extraction is a thinningrelated operation whose central idea is to obtain the locus of the centers of all great circles inscribed in the object or region.

\section{Experiments}

In the following section, the CFAR algorithm proposed in this paper will be used to realize ship detection on the above SAR dataset [11], and the detection results will be compared with those of the traditional two-parameter CFAR algorithm [20] for evaluation research.

\subsubsection{Evaluation Metrics}

In this paper, a large-scale remote sensing SAR dataset [11] is selected for experimentation. With regards to this, three groups of high-resolutiovn SAR images, including Sentinel and Gaofen, are selected for the detection of ship targets. It is detected with a two-parameter CFAR detection method based on Rayleigh distribution, and the false alarm rate established during detection is set to 0.04 . To evaluate the quality and performance of target detection, the Figure of Merit (FoM) detection quality factor is defined as a measure of the effect of ship target detection on high resolution SAR images [21]. Its range is 0 to 1 , the larger the value, the better the quality and effect.

$$
\text { FoM }=\frac{N_{t t}}{N_{g t}+N_{f a}}
$$

where $N_{t t}$ are correct detection targets in the detection, calculated according to the result in the image; $N_{g t}$ are actual targets, obtained based on prior knowledge from SAR images; $N_{f a}$ are false alarm targets, calculated according to the image of the detection result.

Table 1. The running time of the proposed method for a single test image of given size. The running time is tested on a NVIDIA GeForce GTX 960 with the batch size of 1 .

\begin{tabular}{lcc}
\hline Dataset & Image size (pixel) & Running time (s) \\
\hline SAR Ship Dataset & $256 \times 256$ & 2.7274 \\
\hline
\end{tabular}

\subsubsection{Implementation process}

Sea conditions vary intensely according to different climate conditions, so the effect of waves and noise presented in SAR images is also extremely different [21]. For this reason, this simulation experiment uses a two-parameter CFAR algorithm based on the Rayleigh distribution to verify the ship's target detection capabilities in different clutter environments. Two-parameter CFAR detection sets the target window, protection window and background window, and these slide into the image by a certain distance. The protection window is to prevent the part of the ship in the target window from leaking into the background window [20]. Gets the threshold of this local window by calculating the mean and variance of all pixels in the background window, thus it is considered as a ship the part of the target window higher than the threshold. In this algorithm, the size of the set target is adjusted appropriately according to the input SAR image, and through the specified constant false alarm rate, the detection and segmentation between the ship target and background clutter are performed. 


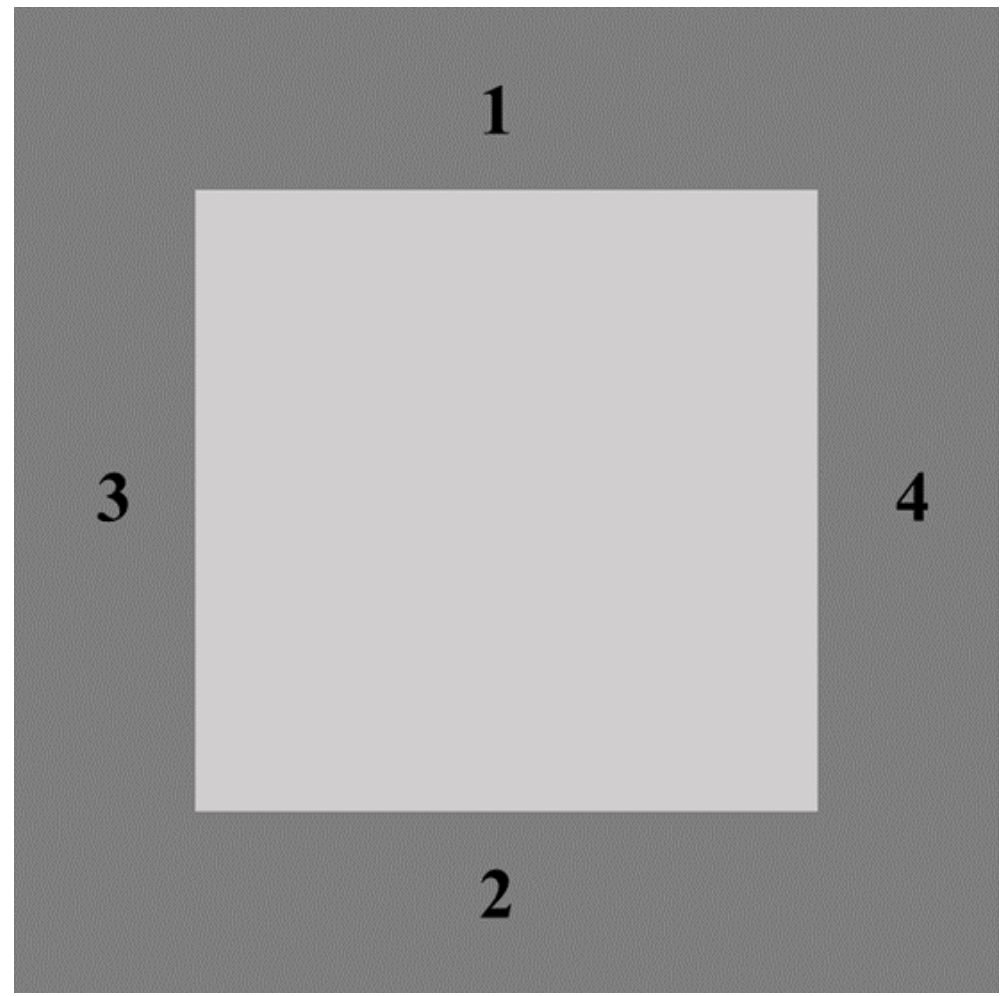

Figure 7. Division of CFAR detection area.

CFAR target detection can be performed after entering the SAR image and determining the CFAR threshold. At this stage, CFAR is divided into four detection areas (clutter estimation areas) which are shown in Figure 7. The detector traverses every pixel in the image and then obtains the mean value and standard deviation according to the corresponding noise area, thus calculating the two-parameter CFAR detection discriminant. According to the result obtained by the discriminant, the discrimination of the target is performed in each pixel, so that a binary image can be obtained.

\subsection{Experimental Results}

Part of the images in the SAR ship dataset are selected to evaluate the algorithm model proposed in this paper. The effect of the double parameter CFAR detector based on the Rayleigh distribution has met expectations. As the cases are shown in Figure 8 of the CFAR segmentation, the target pixels and the background noise pixels are clearly separated, and ship target detection is initially achieved. Especially in the background of uniform sea clutter and noise, the image segmentation effect of ship targets is better. In land and sea cases, the detector also segments ship targets, but the processing of ground noise appears to be the same as that of ships. In most cases, there is a lot of background clutter and false alarms in CFAR detection results, which must be filtered by filtering or MM to obtain detection results. Figure 9 shows the marking result of the ship detection, the image targets are marked as correctly detected targets and false alarm targets by red and yellow frame respectively, the blue is marked as a target whose ship shape is distorted (such as broken) and counted as being within the correct detection target range, and the missed targets are marked with white circles. It can be seen that the two-parameter CFAR detection algorithm based on the Rayleigh statistical model can detect the ships contained in the SAR images. For uniform background situations, false alarm noise can be filtered through filtering and morphological operations to obtain the final detection results; for complex background situations, although all boats are detected, some false alarm targets will still appear in the final results. 


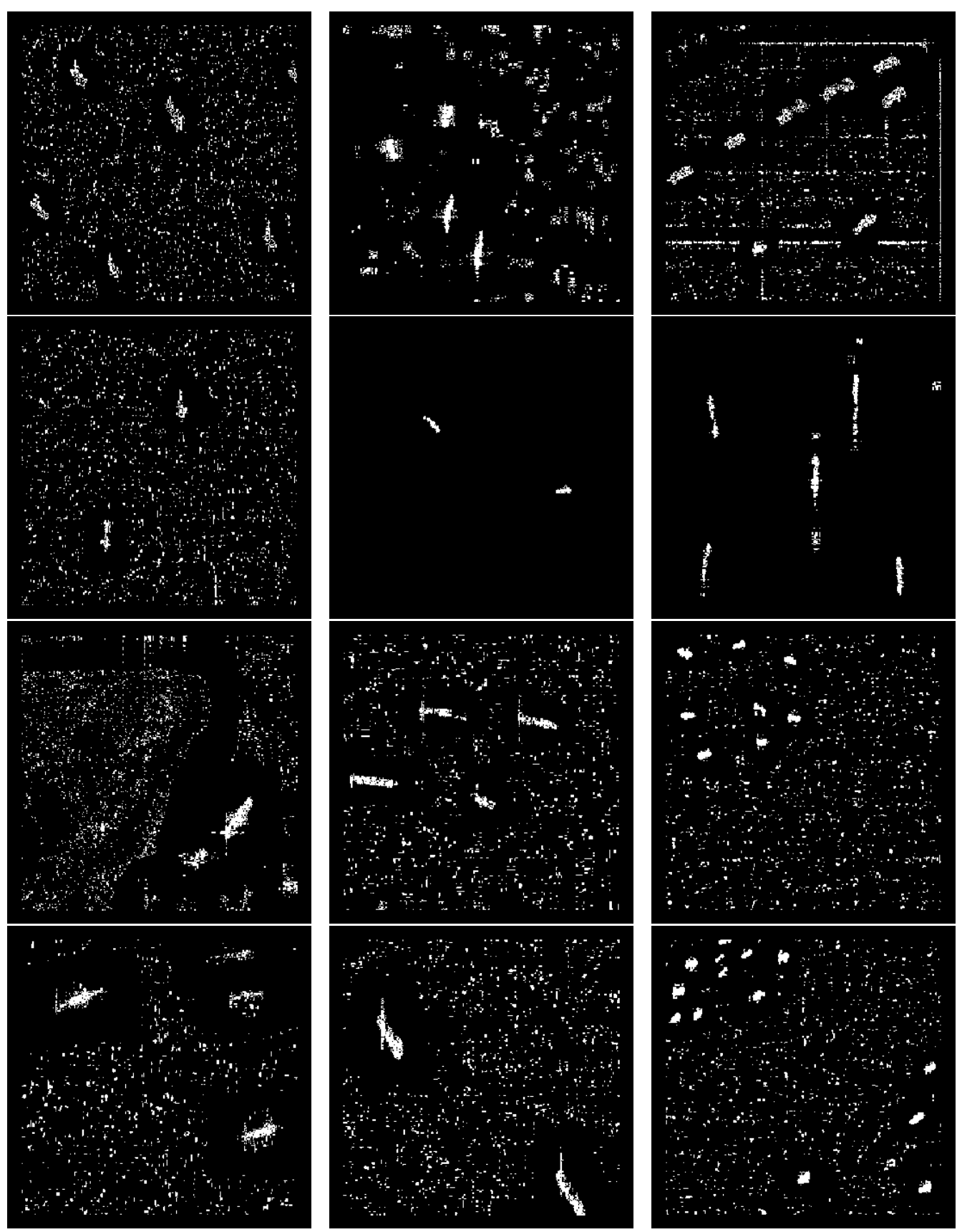

Figure 8. Visualization of the segmentation results. The target and background in each image are clearly distinguished, indicating that the task of segmentation is successful. 


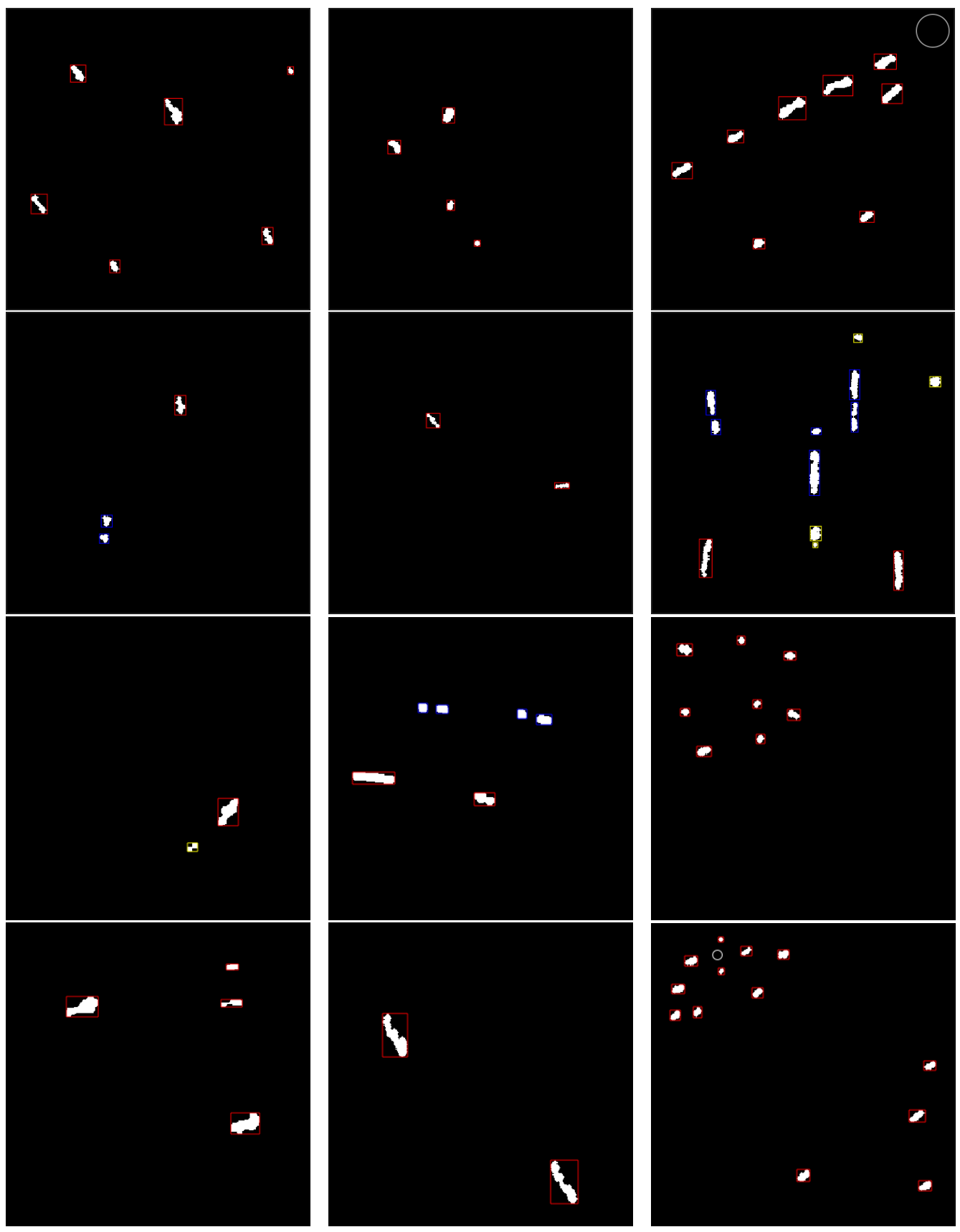

Figure 9. Visualization of the detection results. The detected boxes are shown with different colors or shapes according to different states of the target.

In the above cases, regardless of the Gaofen or Sentinel satellite, the detector has shown promising detection results. But under the background of complicated land and sea conditions, the correct detection rate of the algorithm suffers and there are certain false alarm targets. This is because the statistical clutter model of land and sea conditions does not match the used probability model, leading to target recognition failure. Figures 8 and 9 respectively show some experimental samples and corresponding ship detection results. Therefore, three sets of representative experimental data under different backgrounds were selected for evaluation, namely calm sea, noisy sea and land-sea conditions. Table 2 shows the detection effect and running time of each group under the conditions of experimental images of a given size. The CFAR algorithm proposed in this paper achieves a higher accuracy rate, with an average correct detection rate of $97.1 \%$. It can be seen that the proposed method achieves a higher detection 
accuracy. Across the three groups of evaluations, whether the shape of the ship target is distorted also needs to be noticed. In the task of SAR target detection and recognition, it is necessary to ensure that the target shape is not distorted or broken as much as possible, which will help improve the detection efficiency and target recognition accuracy.

Table 2. Quality index of ship target detection under different sea conditions. The running time is average time for a single image to perform the task.

\begin{tabular}{lccccc}
\hline Background & $N_{t t}$ & $N_{f a}$ & $\begin{array}{c}\text { Number of } \\
\text { missed targets }\end{array}$ & FoM & Running time (s) \\
\hline Calm sea & 21 & 3 & 0 & 0.875 & 2.8765 \\
Noisy sea & 47 & 1 & 1 & 0.959 & 2.4038 \\
Land and sea & 35 & 13 & 2 & 0.700 & 2.9020 \\
\hline
\end{tabular}

The traditional two-parameter CFAR algorithm is based on the Gaussian distribution of noise background [22]. Through the previous experimental results of Ai et al. [20], it can be found that the detection quality of traditional algorithms is not satisfactory in calm seas and wavy seas. The SIR-C image with calm sea (Figure 10 (a)) and the RADARSATSAT SAR image with wavy sea (Figure $10(\mathbf{b})$ ) were used to realize the comparative experiment. The numbers of actual targets in these two images are 18 and 9.

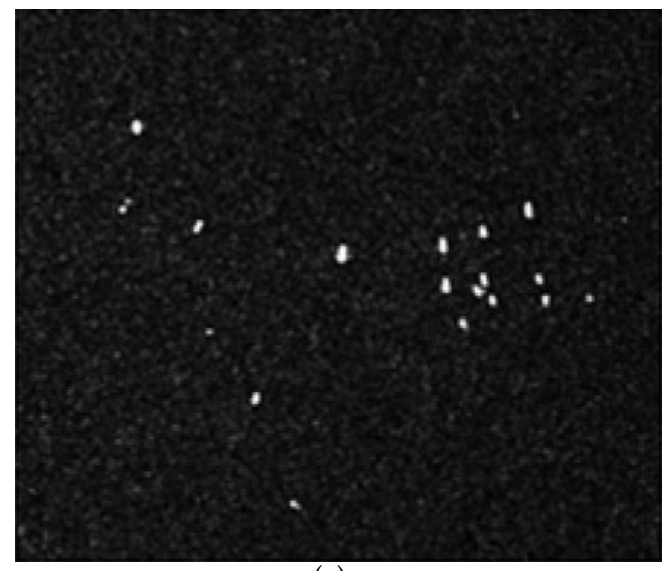

(a)

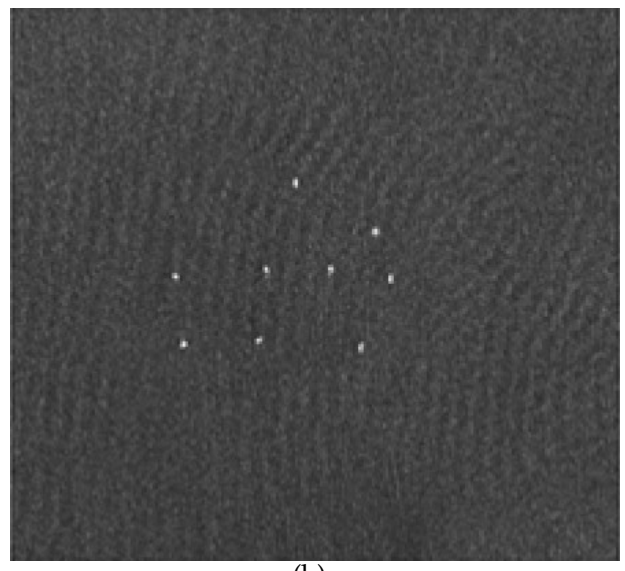

(b)

Figure 10. SIR-C image and RADARSATSAT SAR image.

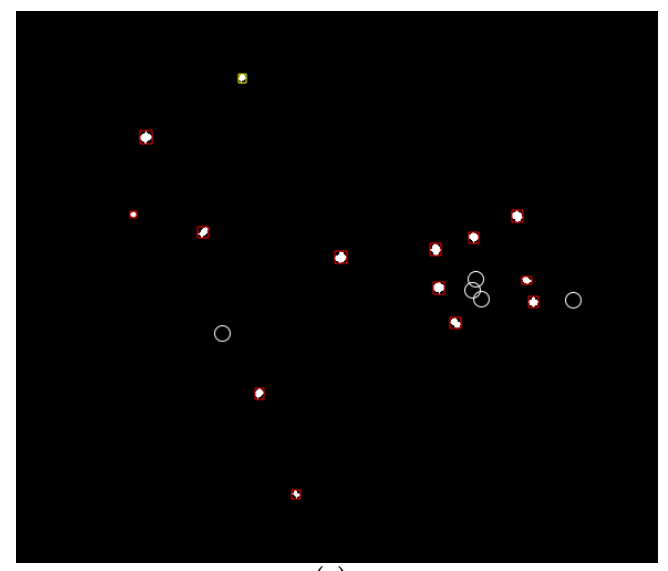

(a)

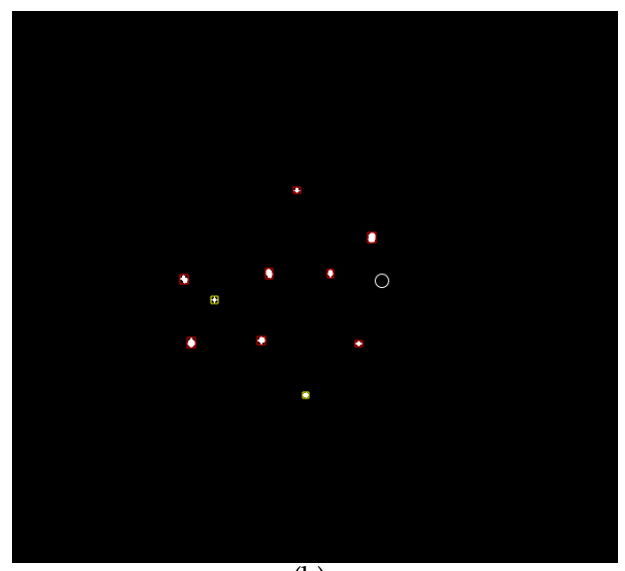

(b)

Figure 11. Visualization of the detection results. Subfigure (a) is the SIR-C image, and (b) is the RADARSATSAT SAR image. 
Table 3. Quality index of ship detection of SIR-C image under different CFAR algorithms. The results of the traditional two-parameter CFAR algorithm are shown in [20].

\begin{tabular}{|c|c|c|c|c|c|}
\hline Background & $N_{t t}$ & $N_{f a}$ & $\begin{array}{c}\text { Number of } \\
\text { missed targets }\end{array}$ & FoM & Running time (s) \\
\hline $\begin{array}{l}\text { Traditional two-parameter } \\
\text { CFAR (Gauss) }\end{array}$ & 13 & 4 & 5 & 0.59 & 0.3750 \\
\hline $\begin{array}{l}\text { Two-parameter CFAR } \\
\text { (Rayleigh) }\end{array}$ & 14 & 1 & 4 & 0.74 & 5.1918 \\
\hline
\end{tabular}

Table 4. Quality index of ship detection of RADARSATSAT SAR image under different CFAR algorithms. The results of the traditional two-parameter CFAR algorithm are shown in [20].

\begin{tabular}{|c|c|c|c|c|c|}
\hline Background & $N_{t t}$ & $N_{f a}$ & $\begin{array}{c}\text { Number of } \\
\text { missed targets }\end{array}$ & FoM & Running time (s) \\
\hline $\begin{array}{l}\text { Traditional two-parameter } \\
\text { CFAR (Gauss) }\end{array}$ & 9 & 8 & 0 & 0.53 & 0.7520 \\
\hline $\begin{array}{l}\text { Two-parameter CFAR } \\
\text { (Rayleigh) }\end{array}$ & 8 & 2 & 1 & 0.73 & 5.4989 \\
\hline
\end{tabular}

The detection results are shown in Tables 3 and 4 . There are missed targets and false alarms in the output results of the traditional two-parameter CFAR algorithm, resulting in a FoM of 0.59 in calm sea and 0.53 in wavy sea. On the contrary, the algorithm of this paper has been greatly improved, and has better results in both cases. The target window size of the traditional two-parameter CFAR algorithm is twice the minimum ship length, so the computation time of this method is relatively faster. The algorithm in this paper needs to traverse each pixel and determine if it is a part of the ship. Therefore, the work takes a little more time and it is necessary to improve computational efficiency. Through the above comparison experiments, it can be seen that the detection effect of the algorithm in this paper is significantly better than the traditional two-parameter CFAR detection algorithm, and it still has great feasibility for ship detection in complex sea conditions.

\section{Discussion}

In the above experiments, the algorithm in this paper has achieved pleasing detection results. Whether it is the target segmentation part or the morphological filtering, the ships are detected and identified. Nevertheless, in addition to physical factors such as the size and shape of the ship, ocean conditions are also a critical factor affecting ship detection capabilities due to the uncertainty of the swell and background noise. When the sea breeze increases, the backscatter from the ocean surface increases, and the brightness contrast between the ship and the ocean surface echo decreases [23,24], which interferes with the algorithm's detection ability to some degree. In follow-up research, the influence of different distribution patterns must be considered, and the appropriate target detection algorithm needs to be investigated for each situation. In addition to image noise, there are many factors such as angle of incidence, ship size, wind speed, sea waves, that affect ship detectability $[25,26]$. Generally, high wind speeds and poor marine environment will cause waves, which make ships surrounded by quite a tough environment. In this paper, the simulation experiment is mainly applied to the detection of ship targets against the background of noise and waves, and the model of these obeys the Rayleigh distribution. Therefore, it is necessary to investigate suitable target detection algorithms for other types of background disorder.

Aiming at the target deformation problem identified in the detection algorithm, the Harris corner detection or sea zone division algorithm can be tried to apply. In this 
way, the ship can be roughly positioned, thus avoiding distortion of the target shape and target breakage. For the problem with the complex background of land and sea, segmenting the SAR image prior to targeting can be considered. The radar image is filtered to separate the sea and land area in the image, which can accurately identify the land. The precision of land-sea segmentation has a direct impact on subsequent target detection missions, so it is necessary to consider how to perfectly eliminate the terrain so that the coastline does not become a false alarm target.

\section{Conclusions}

This paper proposes an improved two-parameter CAFR target detection algorithm based on Rayleigh distribution, combined with mathematical morphological filtering to achieve ship detection based on SAR images. The simulation experiments have been performed to evaluate the capabilities of the algorithm used by the SAR Ship Dataset, and achieved well expected results. In order to address the target segmentation problem of the CFAR algorithm, the morphological operations are integrated, effectively suppressing wave interference and background noise. The target detection effect of the algorithm is acceptable, and the method of MM also shows a great advantage in target recognition. Ships in a complex background are multiscale and relatively small. Currently, there are some computer vision jobs related to the detection of small targets. The main component is a multi-scale function with high resolution, which can improve detection accuracy [27-30]. Considering various types of ships, the distribution model can also be combined with deep learning algorithms for ship detection and recognition. Target detection and recognition is an important topic, and the algorithm still needs continuous improvement. Continuous study of target detection is needed in complex scenes, SAR and target characteristics should be investigated in depth to select and develop the most appropriate probability distribution model.

Author Contributions: It is a single-authored paper. The author has done everything for the paper.

Data Availability Statement: All the SAR images used in this study can be downloaded in CAESAR-Radi at https:/ / github.com/CAESAR-Radi/SAR-Ship-Dataset.

Acknowledgments: The author is very grateful to the team of Researcher Wang, Chao from the Key Laboratory of Digital Earth, Academy of Aerospace Information, Chinese Academy of Sciences for the open source SAR image ship detection data set for providing usable data for this paper.

Conflicts of Interest: The authors declare that there is no conflict of interest regarding the publication of this article.

\section{References}

1. Moreira, A.; Prats-Iraola, P.; Younis, M.; Krieger, G.; Hajnsek, I.; Papathanassiou, Konstantinos P. A tutorial on synthetic aperture radar. IEEE Geoscience and Remote Sensing Magazine 2013, 1, pp. 6-43.

2. Moreira, A.; Krieger, G.; Hajnsek, I.; Papathanassiou, K.; Younis, M.; Lopez-Dekker, P.; Huber, S.; Villano, M.; Pardini, M.; Eineder, M.; De Zan, F.; Parizzi, A. Tandem-L: A Highly Innovative Bistatic SAR Mission for Global Observation of Dynamic Processes on the Earth's Surface. IEEE Geoscience and Remote Sensing Magazine 2015, 3, pp. 8-23.

3. Wu, R. Detección y reconocimiento de blancos basados en imágenes SAR. M.Sc. Thesis, Universitat Politècnica de València, Spain, 2021. http:/ /hdl.handle.net/10251/166986

4. El-Darymli, K.; McGuire, P.; Power, D.; Moloney, C.R. Target detection in synthetic aperture radar imagery: a state-of-the-art survey. Journal of Applied Remote Sensing 2013, 7, pp. 1-35.

5. Oliver, C.; Quegan, S. Understanding Synthetic Aperture Radar Images; SciTech Publ.: Chennai, India, 2004.

6. $\mathrm{Gu}, \mathrm{X}$.; Fu, K.; Qiu, X. Basics of SAR Image Interpretation; Science Press: Beijing, China, 2017.

7. Ouchi, K. Current Status on Vessel Detection and Classification by Synthetic Aperture Radar for Maritime Security and Safety. In Proceedings of the 38th Symposium on Remote Sensing for Environmental Sciences, Gamagori, Aichi, Japan, 3-5 September 2016; pp. 5-12.

8. Wu, L. SAR Image Processing and Target Recognition; Beijing Book Co. Inc.: Beijing, China, 2013.

9. Wackerman, C.C.; Friedman, K.S.; Pichel, W.G.; Clemente-Colón, P.; Li, X. Automatic Detection of Ships in RADARSAT-1 SAR Imagery. Canadian Journal of Remote Sensing 2001, 27, pp. 568-577. 
10. Serra, J. Image Analysis and Mathematical Morphology; Academic Press, Inc.: Orlando, United States, 1983.

11. Wang, Y.; Wang, C.; Zhang, H.; Dong, Y.; Wei, S. A SAR Dataset of Ship Detection for Deep Learning under Complex Backgrounds. Remote Sensing 2019, 11, 765.

12. Rohling, H. Radar CFAR thresholding in clutter and multiple target situations. IEEE transactions on aerospace and electronic systems 1983, 4, pp. 608-621.

13. Lin, J. Parameter estimations of Rayleigh distribution. Mathemtical Quarterly 2000, 15, pp. 49-54.

14. Jakeman, E.; Pusey, P. A model for non-Rayleigh sea echo. IEEE Transactions on Antennas and Propagation 1976, 24, pp. 806-814.

15. Chong, J.; Zhu, M. Target detection algorithm of sar image based on local window K-distribution. Journal of Electronics and Information Technology 2003, 25, pp. 1276.

16. Chen, S.; Li, X. A new CFAR algorithm based on variable window for ship target detection in SAR images. Signal, Image and Video Processing 2019, 13, pp. 779-786.

17. Lin, I-I; Kwoh, L.K.; Lin, Y.;Khoo, V. Ship and ship wake detection in the ERS SAR imagery using computer-based algorithm. In IGARSS'97. 1997 IEEE International Geoscience and Remote Sensing Symposium Proceedings. Remote Sensing - A Scientific Vision for Sustainable Development, Singapore, 1997; pp. 151-153.

18. Liu, Y.; Tang, G.; Zhao, X. Application of Mathematical Morphology in Sea Surface Ship Detection. Periodical of Ocean University of China 2005, 35, pp. 511-514.

19. Ruiz Fernández, L.Á. Aplicación de filtros morfológicos en imágenes. 2020. http://hdl.handle.net/10251/145903

20. Ai, J. Improved two parameter CFAR ship detection algorithm in SAR images. Journal of Electronics and Information Technology 2009, 31, pp. 2881-2885.

21. Robertson, N.; Bird, P.; Brownsword, C. Ship surveillance using RADARSAT scan SAR image. In Alliance for Marine Remote Sensing (AMRS) Workshop on Ship Detection in Coastal Waters, NS, Canada, 31 May 1-2 June 2000; pp. 41-45.

22. He, Y.; Guan, J.; Peng Y.; Lu, D. Radar Automatic Detection and CFAR Processing; Beijing: Tsinghua University Press: Beijing, China, 1999.

23. Chen, D.; Tu, G. A New Algorithm of Ship Targets Detection in SAR Images Using Wavelet Transform. Journal of Electronics and Information Technology 2007, 29, pp. 855-858.

24. Eldhuset, K. An automatic ship and ship wake detection system for spaceborne SAR images in coastal regions. IEEE Transactions on Geoscience and Remote Sensing 1996, 34, pp. 1010-1019.

25. Tings, B.; Bentes, C.; Velotto, D.; Voinov, S. Modelling ship detectability depending on TerraSAR-X-derived metocean parameters. CEAS Space Journal 2019, 11, pp. 81-94.

26. Wang, Y.; Wang, C.; Zhang, H.; Dong, Y.; Wei, S. Automatic Ship Detection Based on RetinaNet Using Multi-Resolution Gaofen-3 Imagery. Remote Sensing 2019, 11, pp. 531.

27. Hu, G.; Yang, Z.; Hu, L.; Huang, L.; Han, J. Small Object Detection with Multiscale Features. International Journal of Digital Multimedia Broadcasting 2018, 2018, pp. 1-10.

28. Cui, L.; Ma, R.; Lv, P.; Jiang, X.; Gao, Z.; Zhou, B.; Xu, M. MDSSD: multi-scale deconvolutional single shot detector for small objects. arXiv preprint arXiv:1805.07009 2018.

29. Guan, L.; Wu, Y.; Zhao, J. SCAN: Semantic Context Aware Network for Accurate Small Object Detection. International Journal of Computational Intelligence Systems 2018, 11, pp. 951-961.

30. Li, J.; Liang, X.; Wei, Y.; Xu, T.; Feng, J.; Yan, S. Perceptual generative adversarial networks for small object detection. In Proceedings of the IEEE conference on computer vision and pattern recognition, Honolulu, HI, USA, 21-26 July 2017 ; pp. 1951-1959. 